\title{
Detection of Possible Interstellar Particles by the HITEN Spacecraft
}

\author{
H. Svedhem
}

Space Science Department, European Space Agency, Postbus 299, NL-2200 AG Noordwijk, The Netherlands

\section{R. Münzenmayer}

Fachgebiet Raumfahrttechnik, Technische Universität München, Richard Wagner Str. 18, D-80333 München, Germany

\section{H. Iglseder}

\author{
ZARM, University of Bremen, Postfach 330440, D-28359 Bremen, . \\ Germany
}

\begin{abstract}
The Cosmic Dust experiment on the Hiten spacecraft detected more than 500 events likely to be associated with particle impacts during its three years life in space. An excess of approximately $40 \%$ was observed in the direction of $220^{\circ}$ ecliptic longitude. This direction corresponds well with the direction of the reported interstellar Helium and interstellar dust seen by other experimenters. In absolute number this is about 20 particles. Since this data was taken during three full years and thus most other effects should be averaged out we believe that these particles are of interstellar origin.
\end{abstract}

\section{Introduction}

The Hiten spacecraft carried a Dust experiment (Igenbergs et al. 1991) of the impact ionisation type with capabilities of detecting particles with a mass down to $10^{-14} \mathrm{~g}$, at a relative impact velocity of $10 \mathrm{~km} / \mathrm{s}$. The instrument aperture of $100 \mathrm{~cm}^{2}$ was pointing perpendicular to the spacecraft spin axis and thus scanning the ecliptic plane each revolution. The spacecraft followed highly elliptic orbits with apogees up to 1.5 million $\mathrm{km}$ during the first two years of operation. Most of the time was spent inside the magnetosphere in the tail region but a number of orbits reached outside the magnetopause in the leading (apex) direction with respect to the earth. The third year the spacecraft was orbiting the moon on elliptic orbits with apolunes up to $50000 \mathrm{~km}$. As a result of the large distance to the earth during most of the mission only a very small fraction of the detected impacts should originate from space debris.

Until recently no clear evidence for the presence of interstellar dust particles in the inner solar system has been found. Several authors have shown that solar radiation pressure and electro dynamic forces may play an important role in the way the particles traverse the solar system. Depending on the orientation of the 


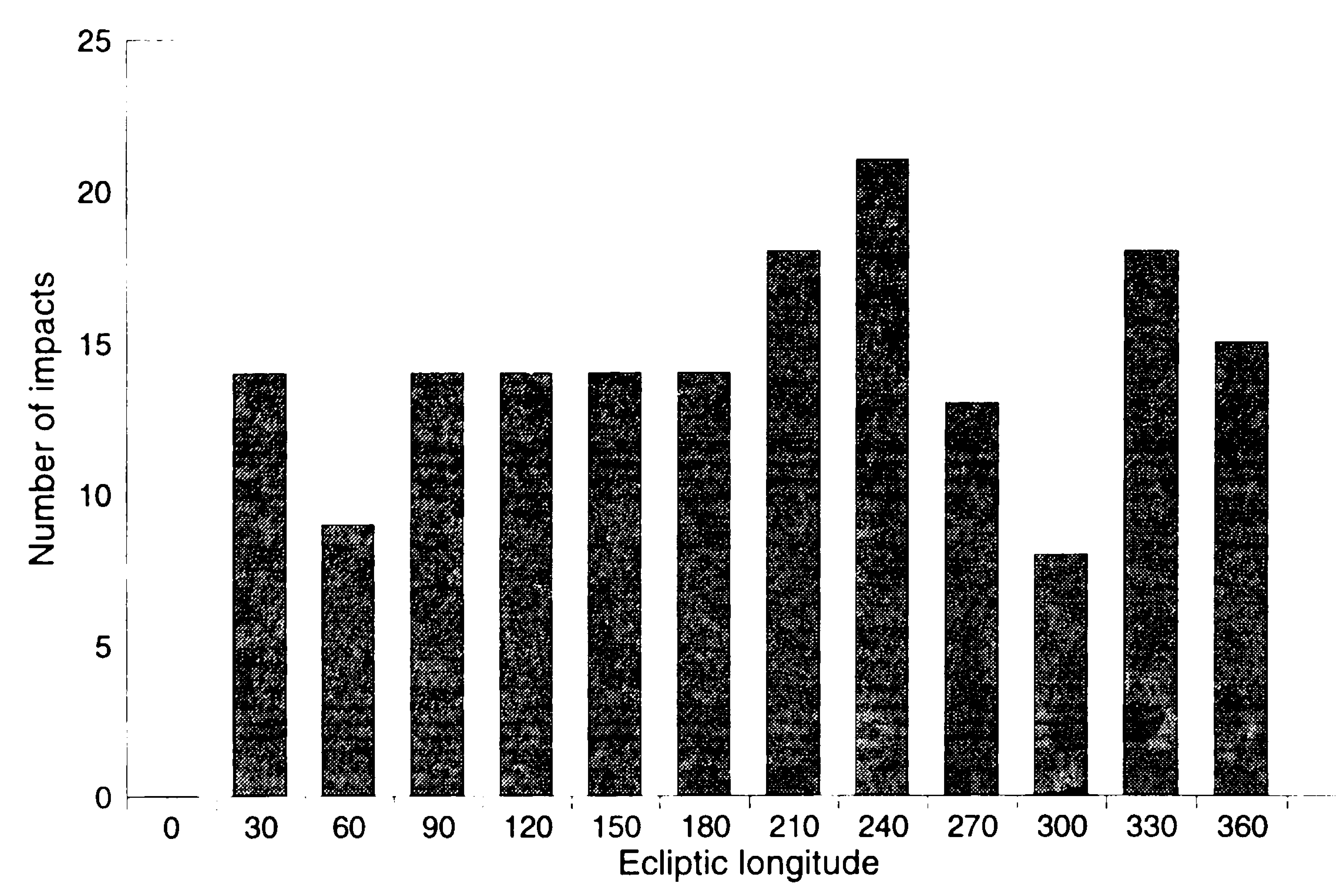

Figure 1. All impacts detected between April 1990 and April 1993 having an impact velocity larger than $15 \mathrm{~km} / \mathrm{s}$, plotted as a function of ecliptic longitude. The bin size, $30^{\circ}$, is approximately equal to half the one dimensional effective field of view of the detector.

solar magnetic field at the time when the particles are entering the heliosphere and the variation of this field during the flight, regions of depletion and enhancement in the number of particles are found (Gustafson \& Misconi 1979, Morfill \& Grün 1979). New results from the Ulysses Dust experiment have shown strong indications for interstellar particles as close as between 3.5 and $6 \mathrm{AU}$ heliocentric distance (Grün et al. 1993, Baguhl et al. 1995).

Now our results show that there is indeed an increase of the flux in the upstream direction of $220^{\circ}$ heliocentric longitude. The absolute number is not very impressive, around 20, due to the small aperture of the instrument but it is still statistically significant thanks to the long exposure time that has resulted in a very stable background flux from other directions.

\section{Observations}

The analysis in this paper is of a statistical nature where the impacts are treated as a set of data and where no conclusions are drawn based on single events. For the calculations only data on spacecraft position, sensor pointing and the measured relative impact velocity has been used. Position and pointing are very accurately known and do not significantly contribute to the uncertainty of the end result. Impact velocity is measured to within a factor $1.6(1 \sigma)$ and is required for the conversion from relative particle direction to ecliptic particle direction. This factor is the most dominant error in the analysis. The uncertainty in the relative particle direction due to the approximately $1 s r$ effective field of view 


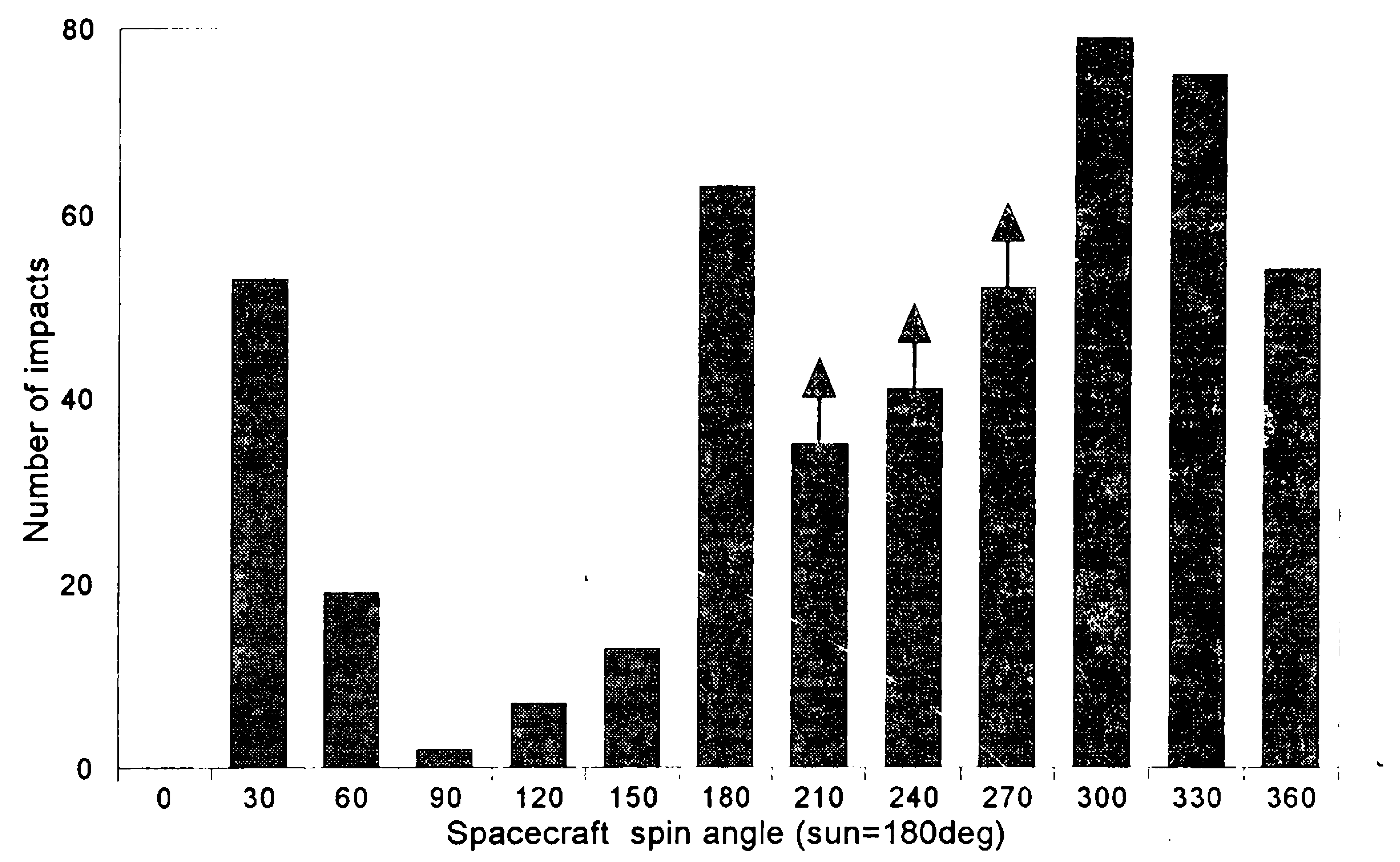

Figure 2. The full set of impacts received by the Hiten detector during its active time of operation between February 1990 and April 1993. The number of impacts are plotted as a function of the spacecraft spin angle referenced to the sun. The sun is fixed at $180^{\circ}$ and the earth's apex direction at $270^{\circ}$.

partly contributes, but mainly as to reduce the angular resolution and it should not be a source of systematic errors.

Figure 1 shows the flux for three full years as a function of ecliptic longitude. A peak around $220^{\circ}$ is clearly visible and possibly a lower peak at $320^{\circ}$. It is of great importance to show these data for an integer number of years since only in this case the earth apex direction has swept through all directions at equal duration. The earth's apex direction strongly favours detection of impacts due to the higher relative velocity. This is clearly visible in Figure 2 where impacts are plotted as a function of a direction referred to the sun and the apex direction. The three arrows indicate that these bins contain less impacts than the experiment probably has received. This is caused by a partly blinding effect, by photo electrons generated by solar photons, during the period of a quarter of each revolution when the sensor is gradually increasing its view of the sun. The peak at spin phase between $150^{\circ}$ and $180^{\circ}$ is clearly from $\beta$-meteoroids on prograde hyperbolic orbits. They can also easily be identified by their much higher velocities than the background flux.

\section{Discussion}

If we assume that the excess at $220^{\circ}$ longitude is of interstellar origin we can calculate a minimum flux, since the number may be higher due to the elimination of particles with impact velocities below $15 \mathrm{~km} / \mathrm{s}$. Taking geometrical factors 
into account a flux of $1.2 \times 10^{-4} \mathrm{~m}^{-2} \mathrm{~s}^{-1}$ is found, indeed close to the $0.8 \times$ $10^{-4} \mathrm{~m}^{-2} \mathrm{~s}^{-1}$ reported from Ulysses (Grün et al. 1993). The mass sensitivity threshold for the two detectors is of the same order.

Results from the Ulysses Neutral Gas Experiment show that interstellar helium arrives from a direction (at infinity) of $252^{\circ}$ longitude at a velocity (at infinity) of $26 \mathrm{~km} / \mathrm{s}$ (Witte et al. 1993) while the Ulysses Dust Experiment found a direction (in situ) of $280^{\circ}$ (Baguhl et al. 1995).

If one compares the velocity distribution between particles arriving from between $180^{\circ}$ and $240^{\circ}$ longitude to the velocity distribution of all particles one will find an excess of particles in the band 20 to $30 \mathrm{~km} / \mathrm{s}$ for the particles arriving from between $180^{\circ}$ and $240^{\circ}$. This seem to indicate that the interstellar particles have not gained much velocity while approaching the sun. This could be explained if the $\beta$ value (defined as the ratio between the force exerted by the solar radiation pressure and the force from the solar gravitation, $F_{r p} / F_{g}$ ) is close to or only slightly less than one. In this case interstellar particles detected during spring, when the earth apex direction is facing the incoming particles, will arrive from a somewhat lower longitude than the longitude at infinity and during fall the opposite will be the case. In addition the probability of detecting these particles during fall will be reduced since the relative velocity is much lower. We do see an enhanced flux at a lower longitude, $220^{\circ}$ (spring?), and a less enhanced flux at higher longitudes, $320^{\circ}$ (fall?), both with an (absolute) velocity of between 20 and $30 \mathrm{~km} / \mathrm{s}$.

One could argue that any increase in the background flux, even if isotropic, would if it occurs during spring appear as an increase of the flux in the direction of the interstellar particles, due to the high sensitivity in the apex direction. We cannot, however, see a reason for a seasonal variation in the natural background flux strong enough to be of importance. There seems, for example, not to be any correlation with known meteor streams. It has been proposed that particles in orbits in resonant lock with the earth could cause a modulation of the background flux, with a maximum in October and a minimum in April (Dermott et al. 1994). This could possibly influence the data at $320^{\circ}$ but not the data the major peak around $220^{\circ}$. Further search for sources of systematic errors and spacecraft activities etc has been resultless.

With this explanation our results at $1 A U$ seem compatible with results from the two other instruments mentioned above. Further analysis, including characterisation of individual impacts, will show if this indeed is the case.

\section{References}

Baguhl, M., et al. 1995, Science, 268, 1016

Dermott, S.F.,et al. 1994, Nature, 369, 719

Grün, E., et al. 1993, Nature, 362, 428

Gustafson, B.A.S. \& Misconi, N.Y 1979, Nature, 282, 276

Igenbergs, E., et al., 1991, in Origin and Evolution of Interplanetary Dust, A.C. Levasseur-Regourd \& H. Hasegawa, Dordrecht: Kluwer, 45

Morfill, G.E. \& Grün, E. 1979, Planet. Space Sci., 27, 1283

Witte, M., et al. 1993, Adv. Space Res., 13, (6)121 